\title{
Validation of Atmospheric InfraRed Sounder (AIRS) Spectral Radiances with the Scanning High-resolution Interferometer Sounder (S-HIS) aircraft instrument
}

\author{
Henry E. Revercomb, David C. Tobin, Robert O. Knuteson, Fred A. Best, \\ William L. Smith*, Paul van Delst, Daniel D. LaPorte, Scott D. Ellington, Mark \\ W.Werner, Ralph G. Dedecker, Ray K. Garcia, Nick N. Ciganovich, H.Benjamin Howell, \\ Steven Dutcher, and Joe K. Taylor \\ University of Wisconsin-Madison, Space Science and Engineering Center \\ 1225 West Dayton Street, Madison Wisconsin, 53706 \\ * NASA Langley Research Center
}

\begin{abstract}
The ability to accurately validate high spectral resolution IR radiance measurements from space using comparisons with aircraft spectrometer observations has been successfully demonstrated. The demonstration is based on a 21 November 2002 under-flight of the AIRS on the NASA Aqua spacecraft by the S-HIS on the NASA ER-2 high altitude aircraft and resulted in brightness temperature differences approaching $0.1 \mathrm{~K}$ for most of the spectrum!
\end{abstract}

Aircraft comparisons of this type provide a mechanism for periodically testing the absolute calibration of spacecraft instruments with instrumentation for which the calibration can be carefully maintained on the ground. This capability is especially valuable for assuring the long-term consistency and accuracy of climate observations, including those from the NASA EOS spacecrafts (Terra, Aqua and Aura) and the new complement of NPOESS operational instruments. The validation role for accurately calibrated aircraft spectrometers also includes application to broadband instruments and linking the calibrations of similar instruments on different spacecraft.

Both the AIRS and the S-HIS calibrations are expected to be very accurate (formal 3sigma estimates are better than $1 \mathrm{~K}$ brightness temperature for a wide range of scene temperatures), because high spectral resolution offers inherent advantages for absolute calibration and because they make use of high emissivity cavity blackbodies as onboard radiometric references. AIRS has the added advantage of a cold space view, and the SHIS calibration has benefited from the availability of a zenith view from high altitude flights on the Proteus aircraft. The S-HIS has also benefited from calibration techniques developed over many years in conjunction with the original HIS aircraft instrument and with the Atmospheric Emitted Radiance Interferometer (AERI) instruments developed for the DOE ARM Program. The absolute radiometric calibration is traceable to NIST, and in the future, we plan to check the calibration directly by inter-comparison to a NISTmaintained sensor (the TXR radiometer).

It is expected that aircraft flights of the S-HIS and its close cousin the NPOESS Atmospheric Sounder Testbed (NAST) will be used to check the long-term stability of AIRS and the NPOESS operational follow-on sounder, the Cross-track Infrared Sounder (CrIS), over the life of the mission. 


\section{Introduction}

The need for higher accuracy and more refined error characterization of radiance measurements from space (and corresponding geophysical products) to improve both weather forecasting and climate change monitoring has led to a new emphasis on conducting direct tests of in-orbit performance, referred to as "validation". Validation involves collecting higher quality reference data from specially maintained airborne and ground-based facilities that can support refined analyses of a controlled set of wellunderstood measurements, instead of statistical analyses of data having inconsistent pedigree and unknown error characteristics. This is a positive trend that will help take full advantage of our satellite systems.

The validation activities initiated for the NASA Earth Observing System (EOS) platforms, which are well underway for the Terra and Aqua platforms and will soon be initiated for Aura, are setting the stage for enhanced validation of new observational satellite systems. Extensive plans for validation of the National Polar Orbiting Environmental Satellite System (NPOESS) and future geosynchronous systems are also underway. The Scanning High-resolution Interferometer Sounder (S-HIS) aircraft instrument discussed in this paper is an important validation tool that is currently being used for both EOS and NPOESS. For NPOESS, S-HIS use is coordinated with the NPOESS Airborne Sounder Testbed (NAST) to optimize payload compatibility with joint field campaigns and for critical inter-comparison tests of accuracy.

\section{The Scanning High-resolution Interferometer Sounder (S-HIS)}

The Scanning HIS is an airborne Michelson Interferometer follow-on to the original University of Wisconsin HIS (Smith, et al., 1989; Revercomb, et al., 1988a) that was flown successfully on the NASA ER2 from 1986 to 1998. The original nadir-only spatial sampling of the HIS has been replaced by cross-track coverage with similar 2-km footprints, while at the same time S-HIS is smaller and much easier to operate (Revercomb, et al., 1996, 1998). In addition to the NASA ER2, it has been successfully flown on the NASA DC8 and on the Scale Composites Proteus, as shown in Figure 1. As illustrated, the Proteus implementation also provides a zenith view to augment calibration information and as a valuable capability for studying upper level water vapor.

Typical S-HIS radiance spectra are shown in Figure 2. The overlapping regions of the three spectral bands are used to constrain the non-linearity correction required for the longwave and midwave bands that use photo-conductive HgCdTe detectors. The shortwave band uses an InSb detector and its expected linearity is confirmed by the lack of telltale out-of-band contributions. As with the original HIS (Revercomb, et al., 1988b, 1989, 1997; Best, et al., 1997), accurate calibration has been a major goal for the S-HIS. Figure 3 shows the 3-sigma radiometric performance expected for S-HIS. Note that for scene temperatures above 220 to $240 \mathrm{~K}$, the 3-sigma absolute calibration accuracy is less than $0.3 \mathrm{~K}$ ! For carefully calibrated IR spacecraft instruments, the accuracy will be even better for cold scene temperatures, because space provides a well-known cold reference.

The zenith viewing option was first implemented in November 2002. Up and downlooking observations from $14 \mathrm{~km}$ altitude are shown in Figure 4. Note the accurate zero determined from the warm and intermediate temperature blackbody spectra shown, even for the non-linear longwave and midwave bands. The zenith view adds an important constraint on the S-HIS non-linearity correction algorithm (Revercomb, 1994). 

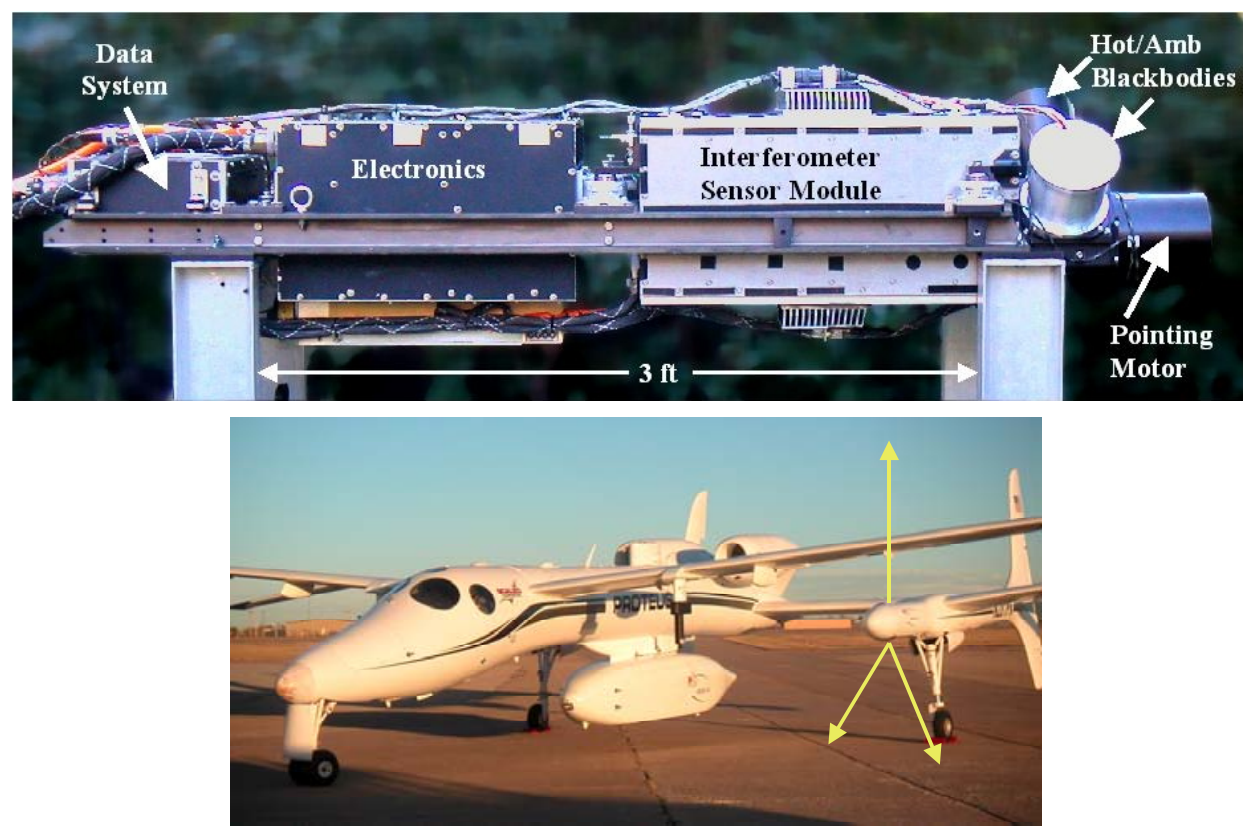

Figure 1. Scanning HIS instrument and illustration of cross-track and zenith viewing from the Proteus aircraft. S-HIS has been configured to fly on the NASA DC8 and ER2 aircraft as well as the Proteus.
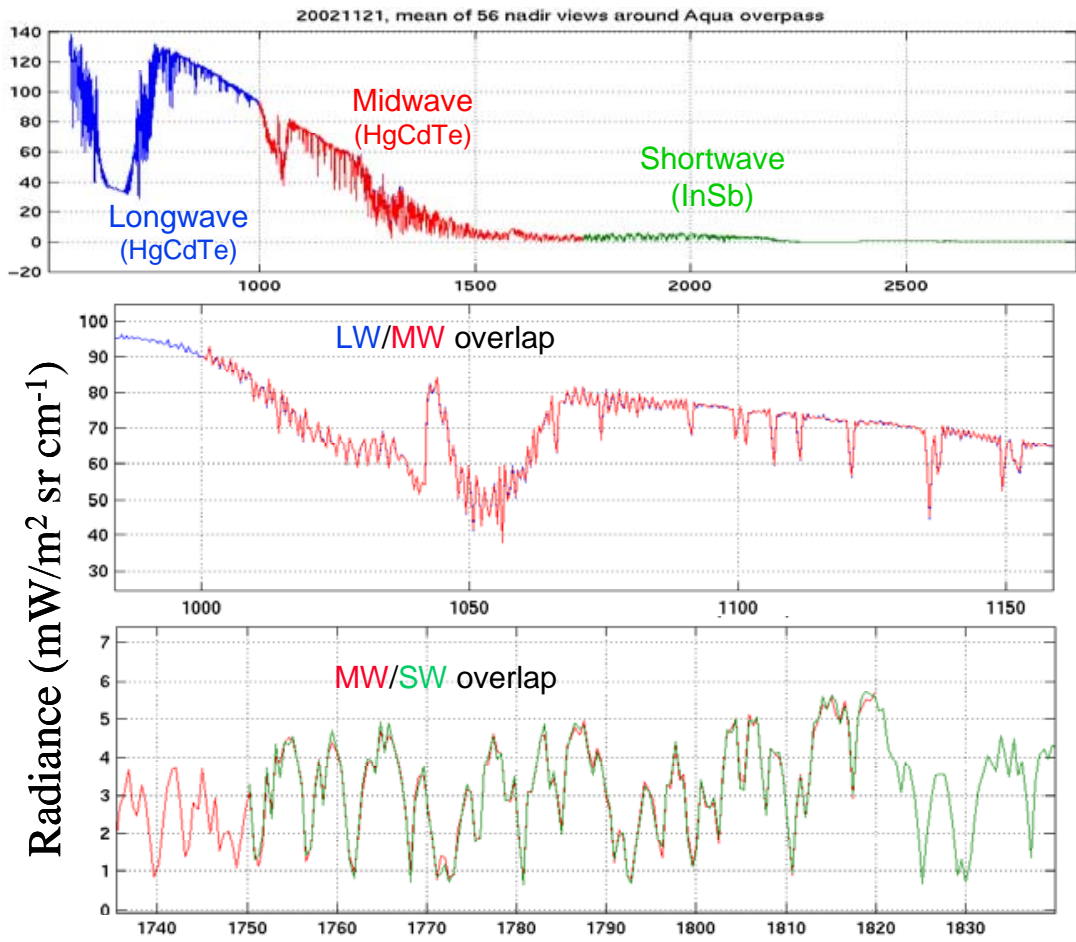

Figure 2. Scanning HIS radiance spectra collected over the Gulf of Mexico on 21 November 2002 from the NASA ER2 at $20 \mathrm{~km}$ altitude during the Terra-Aqua Experiment (TX-2002). The three separate spectral bands of S-HIS are illustrated, along with the good agreement between spectra from neighboring bands where they overlap. 

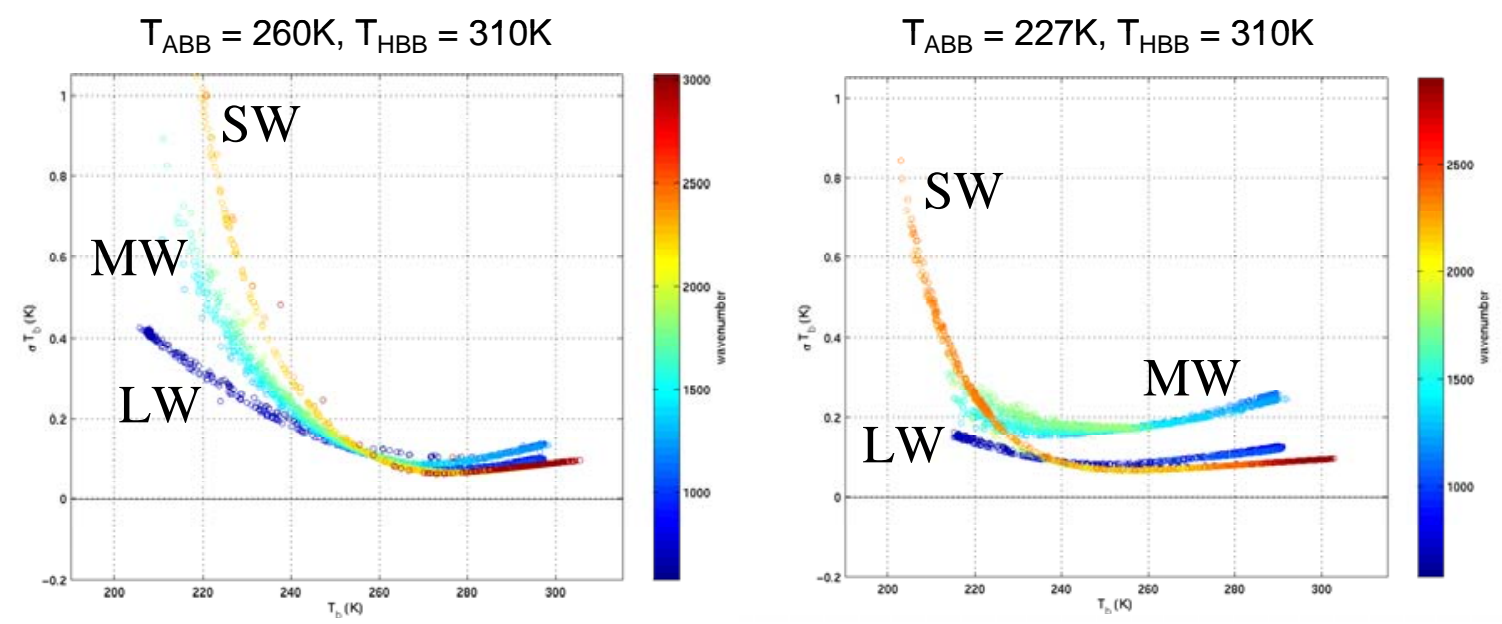

Figure 3. Three-sigma calibration accuracy estimates for Scanning HIS with two different ambient blackbody temperatures. Conditions apply to AIRS vadiation flights on 21 November 2002 on the ER2 over the Gulf of Mexico (left) and 16 November 2002 on Proteus over the DOE Atmospheric Radiation Measurement (ARM) site in Oklahoma. $\mathrm{T}_{\mathrm{ABB}}$ and $\mathrm{T}_{\mathrm{HBB}}$ are the ambient and hot blackbody temperatures.

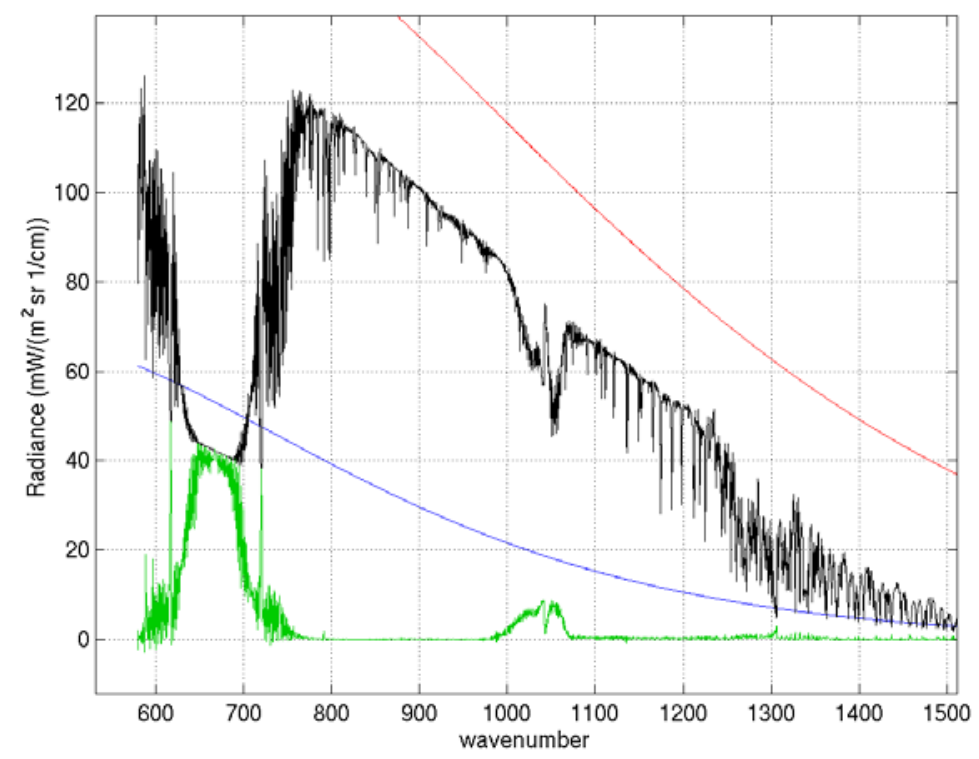

Figure 4. Comparison of zenith (green) and nadir (black) radiance spectra from the Proteus at $14 \mathrm{~km}$ over the DOE Atmospheric Radiation Measurement (ARM) program Southern Great Plains site on 16 November 2002. Spectra of the reference blackbodies used for calibration are shown in red and blue.

\section{Validation of AIRS with Scanning HIS}

The Atmospheric Infrared Sounder (AIRS) on the NASA Aqua spacecraft launched on 20 May 2002 is a cryogenic cross-dispersed grating spectrometer (Aumann et al., 2003). It employs 7 different orders of dispersion to map the spectrum onto 12 detector modules with a total of 15 linear arrays. A single spatial footprint is detected at a time, with the field being focused on the grating to minimize the impact of non-uniform cloudy scenes on spectral calibration. 
The differences in spatial and spectral sampling that need to be accounted for in making accurate comparisons of AIRS and S-HIS are illustrated in Figure 5. The technique selected for doing this is to make use of calculations that account for the actual spectral and spatial characteristics of each instrument. The calculated spectra allow the observation-minus-calculation residual for each instrument to be compared, avoiding the first-order effects of these differences. To improve this comparison even further, the residuals are each convolved with the Instrument Line Shape (ILS) of the other. This is equivalent to eliminating grating contributions from optical path differences larger than measured by S-HIS and weakly apodizing S-HIS to match the effect of the AIRS ILS.
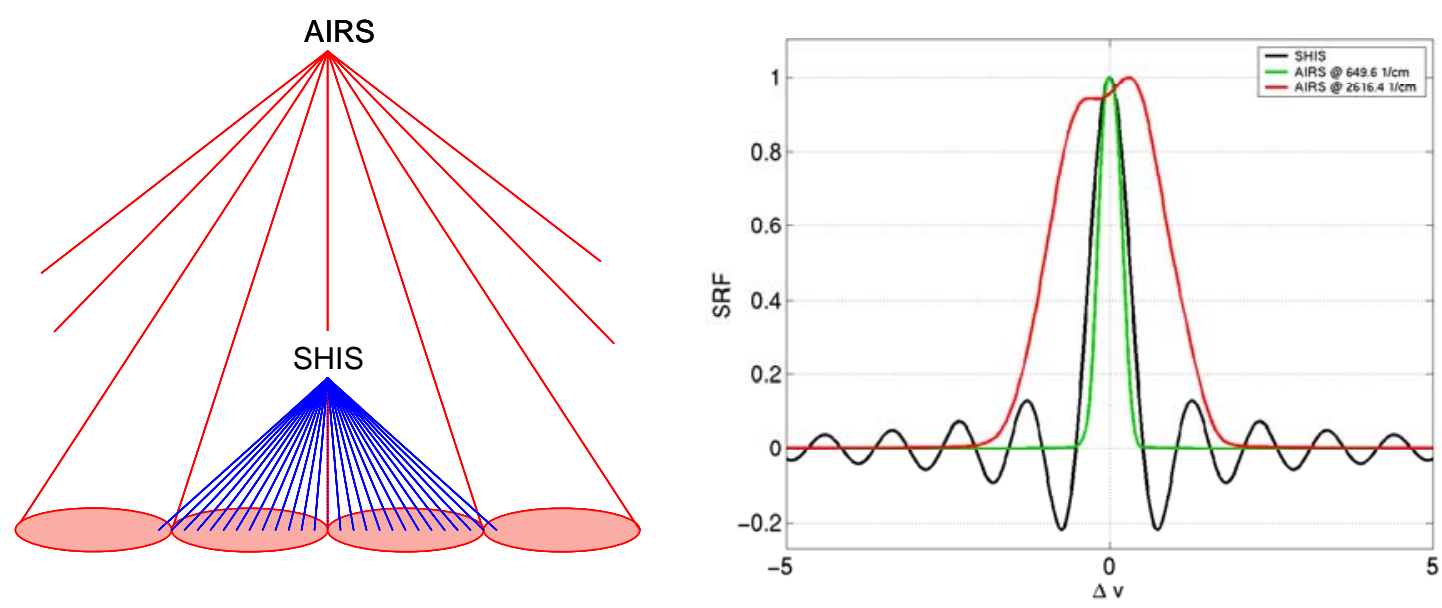

Figure 5. Illustration of the differences between AIRS and S-HIS spatial viewing conditions (left) and spectral Instrument Line Shape (ILS) functions that need to be accounted for in making detailed radiance comparisons.

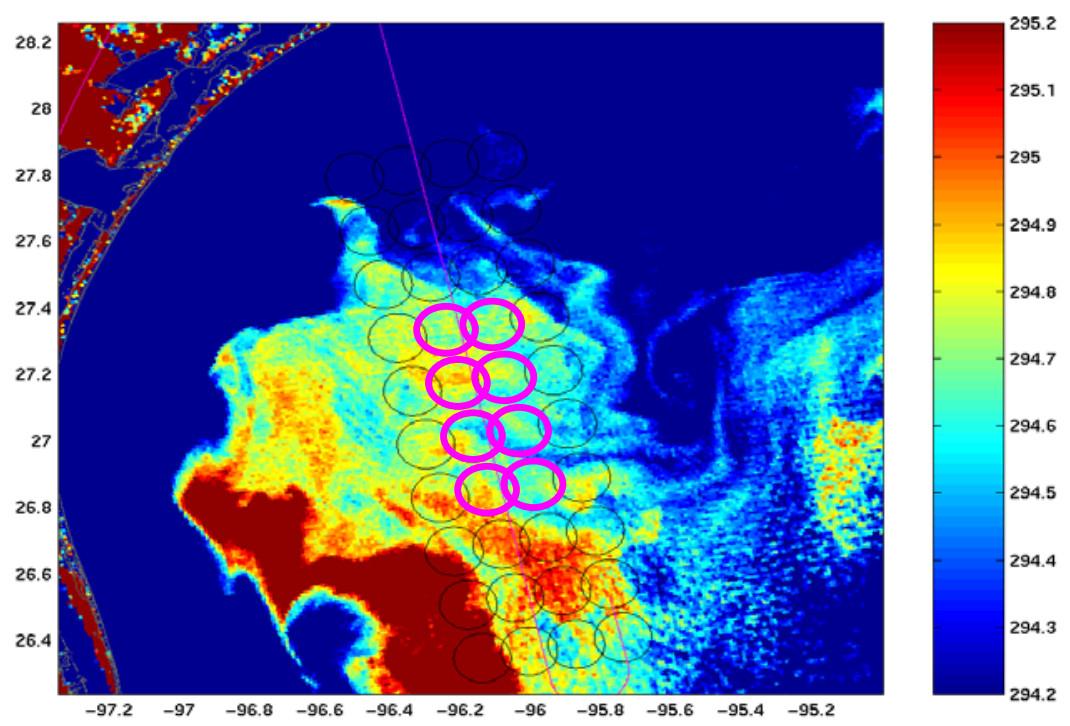

Figure 6. Aqua MODIS 12 micron band image over the Gulf of Mexico on 21 November 2002, illustrating the aircraft sub-track (narrow line) and the 8 near nadir AIRS footprints ( $\sim 15 \mathrm{~km}$ diameter) chosen for comparison based simultaneity and scene uniformity (peak-to-peak brightness temperature variations of about $0.5^{\circ} \mathrm{C}$ ). 
The best conditions for validation of AIRS with Scanning HIS to date were encountered over the Gulf of Mexico on 21 November 2002 as part of the Terra Aqua Experiment (TX 2002). The excellent scene uniformity is illustrated in Figure 6, and the first-order spectral comparison is shown in Figure 7. For this first-order comparison, the spectra from each instrument have been noise filtered using principle component techniques, and averaged over common spatial footprints.
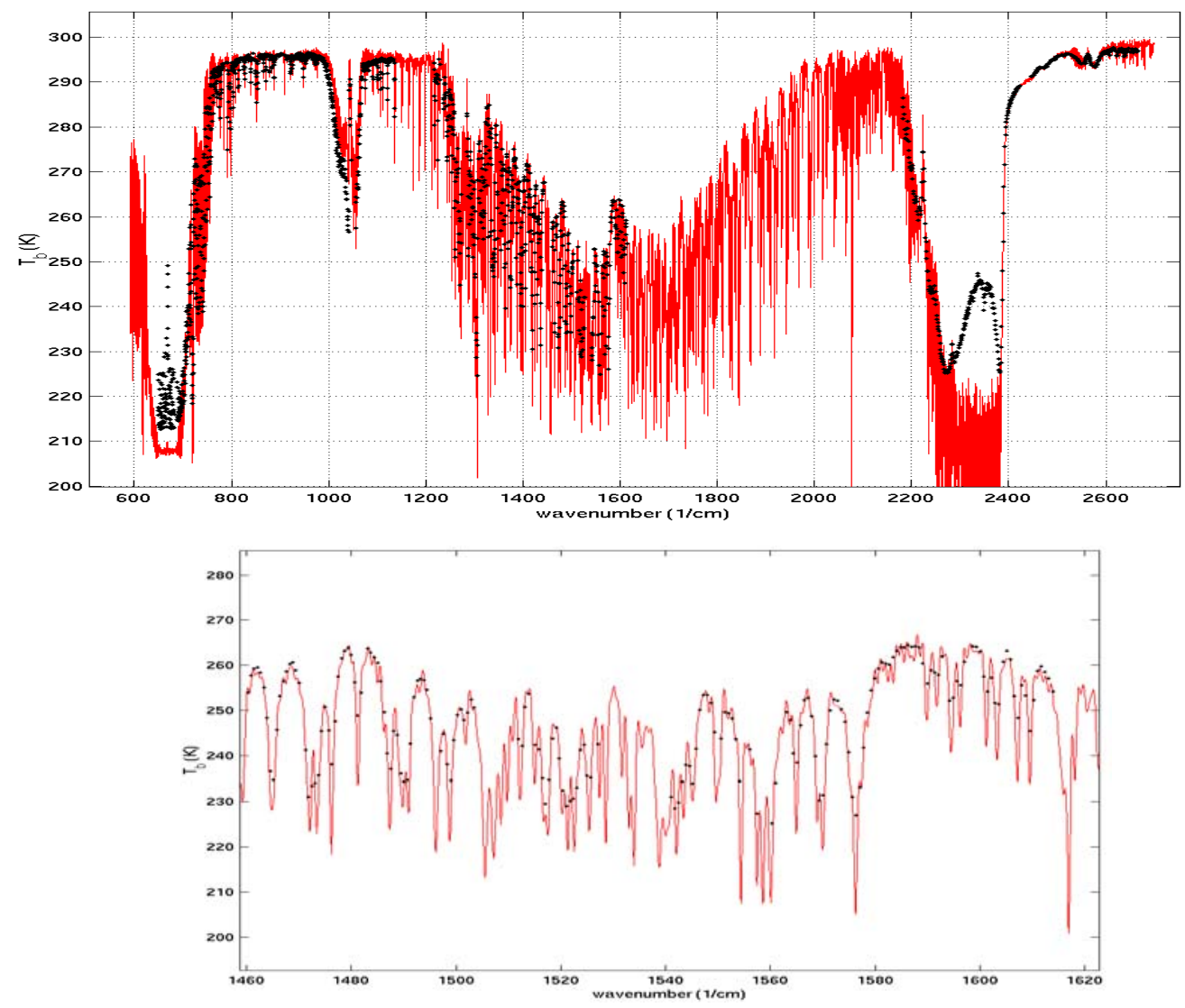

Figure 7. Average of Scanning HIS spectra (red) from inside 8 AIRS footprints (see Figure 6) compared to AIRS spectral channels (black +'s) averaged over the 8 footprints. For this comparison, nothing has been done to account for the different spectral characteristics or altitudes of the two platforms. The portions of the AIRS spectra in the middle of the $\mathbf{1 5}$ and 4-micron carbon dioxide bands are sensitive to altitudes above the ER2 altitude of the S-HIS and should not be expected to agree. However, the spectrum from near the middle of the 6.3-micron water vapor band (lower blow-up) is a region where the effective spectral resolutions are comparable, and where the major contributions come from below the ER2. Note the excellent agreement in this region.

The final comparison between S-HIS and AIRS radiances is shown in Figure 8 as the difference of spectrally normalized obs-calc residuals. The excellence of the agreement is demonstrated by the histograms for each module, as discussed in the caption. Note that while the small residual differences are not just for window regions, but extend deeply into the 15-micron carbon dioxide band and also into the 6.3-micron water vapor band. The analysis also identified a spectral scale error in one AIRS module (Figure 9). 

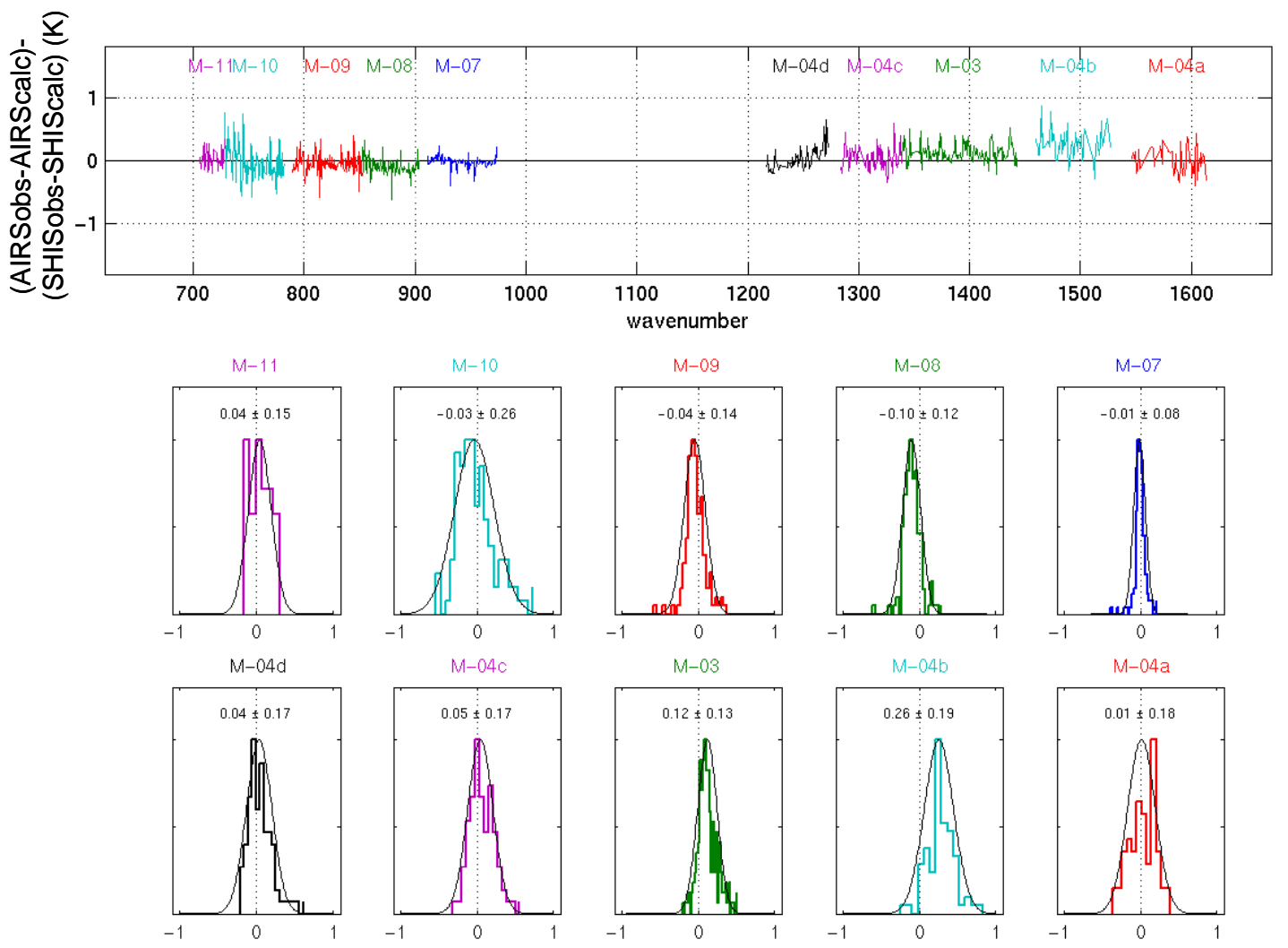

Figure 8. Summary of final AIRS to Scanning HIS comparison with elimination of channels with significant sensitivity above the ER2. The colors distinguish different detector modules of the AIRS instrument (separate linear detector arrays), with corresponding color coded histograms of differences. Note the excellent agreement! The mean agreement over most modules is order $0.1^{\circ} \mathrm{C}$ or less $(\mathrm{M}-04 \mathrm{~b}$ is the one exception with a mean of $0.26{ }^{\circ} \mathrm{C}$ ). Also, the standard deviations are with one exception less than $0.2^{\circ} \mathrm{C}$.

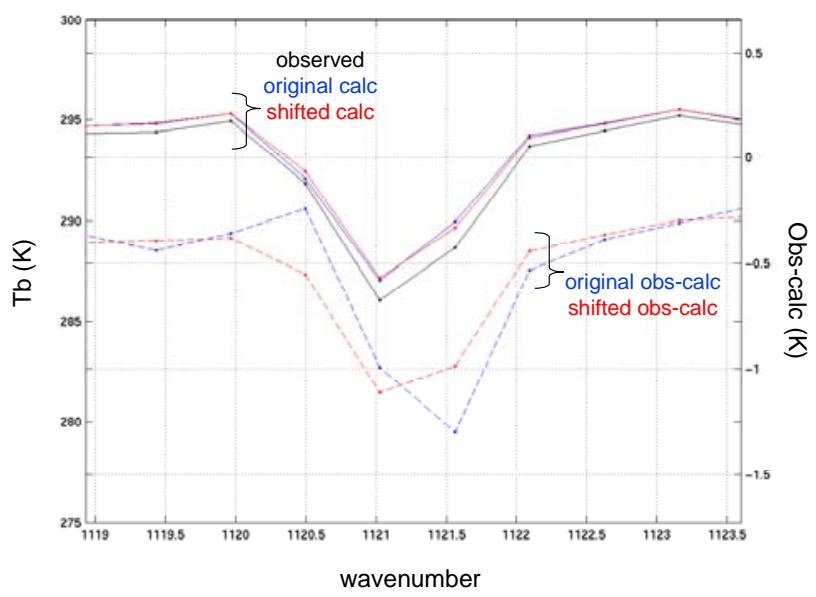

Figure 9. Evidence for very small shift in the spectral calibration of AIRS channels (3\% of spectral resolution) from module M-05 discovered with S-HIS. The original shift has now been confirmed with direct comparisons of calculated spectra with AIRS and the spectral response functions adjusted to remove it. 


\section{Assessment of MODIS Calibration from AIRS}

Having validated AIRS with S-HIS, it is useful to consider the implications for other infrared instruments. Since the AIRS provides reasonably high spectral resolution, it can be used to simulate the radiance of lower resolution instruments, such as the EOS MODIS imager, by convolving the AIRS spectrum with the normalized MODIS spectral resolution function for each of the 15 MODIS IR bands. A sample AIRS spectrum is compared to MODIS spectral response functions in Figure 10. Note that there are some gaps in the AIRS spectrum that create significant convolution errors, unless they are accounted for.
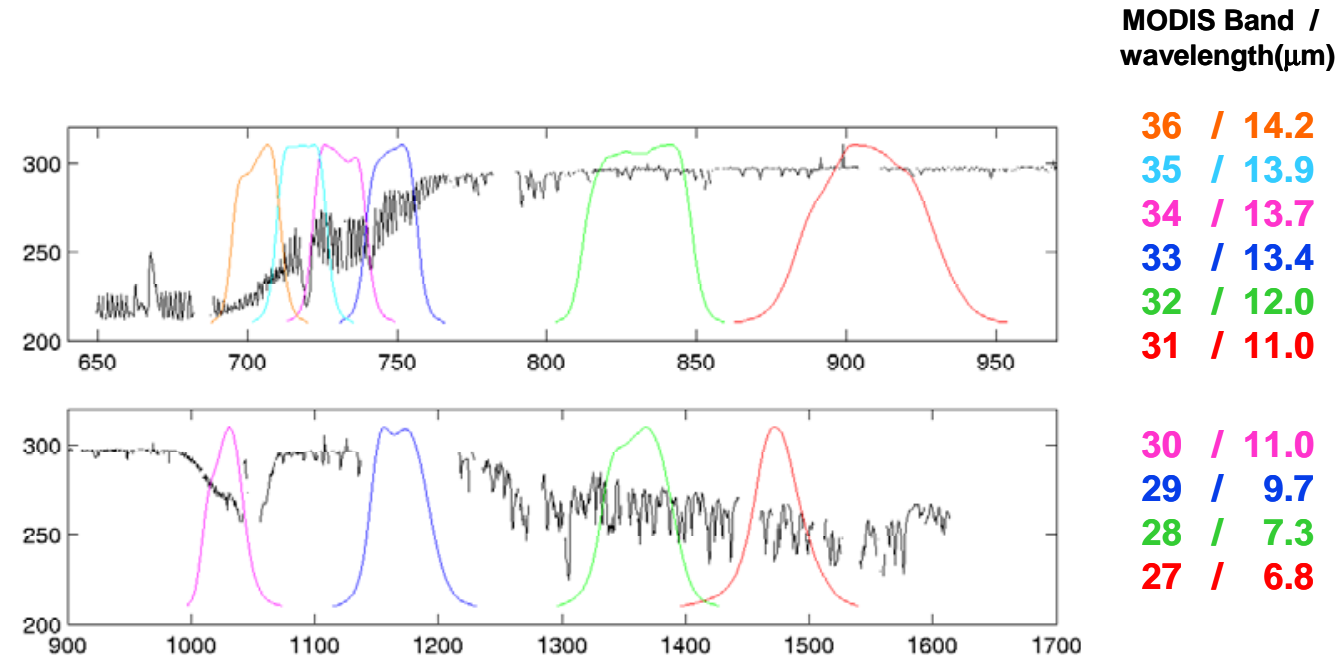

30 | 11.0

29 I 9.7

28 | 7.3

$27 \quad 6.8$

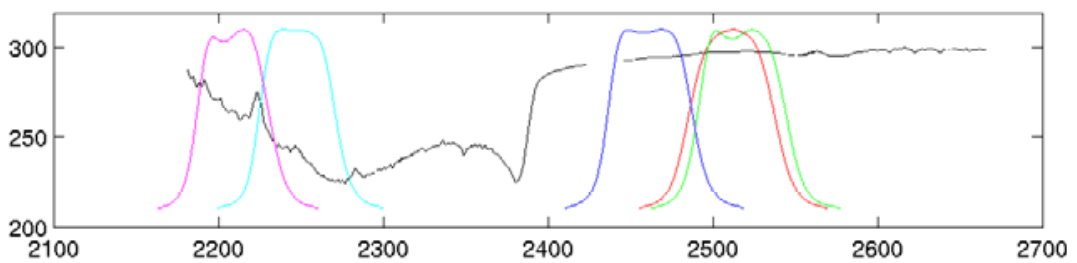

$\begin{array}{lll}25 & I & 4.5 \\ 24 & I & 4.4 \\ 23 & I & 4.1 \\ 22 & I & 4.0 \\ 21 & I & 4.0\end{array}$

Figure 10. Comparison of AIRS spectrum and MODIS spectral response functions for its overlapping IR bands. It is clear that most MODIS bands can be simulated with AIRS by convolving the AIRS spectra with the MODIS spectral response functions.

The results of comparing co-located AIRS and MODIS for a large selection of global samples, chosen from reasonably uniform AIRS footprints is shown in Figure 11. Note that the numbers of comparisons range from a few 100,000 for window channels and the ozone channel to almost 2 million for the most opaque channel 36. The distributions of AIRS-MODIS differences shown in blue for each band are the final results with corrections for convolution errors determined from a standard atmosphere. The blue bars give an estimate of the convolution errors and are centered at the mean AIRS-MODIS difference. Given the excellent agreement between AIRS and S-HIS, it is expected that the largest part of these differences are issues with MODIS. The most significant differences occur for the most opaque 15micron CO2 bands (34-36) and for water vapor $(27,28)$. 
Red=without accounting for convolution error Blue=accounting for convolution error with meal correction from standard atmospheres

\section{p-p Convolution Error (CE) Estimate}

\begin{tabular}{crrrrr} 
Band & Diff & CE & Diff & Std & \multicolumn{1}{c}{$\mathbf{N}$} \\
21 & 0.10 & -0.01 & 0.09 & 0.23 & 187487 \\
22 & -0.05 & -0.00 & -0.05 & 0.10 & 210762 \\
23 & -0.05 & 0.19 & 0.14 & 0.16 & 244064 \\
24 & -0.23 & 0.00 & -0.22 & 0.24 & 559547 \\
25 & -0.22 & 0.25 & 0.03 & 0.13 & 453068 \\
27 & 1.62 & -0.57 & 1.05 & 0.30 & 1044122 \\
28 & -0.19 & 0.67 & 0.48 & 0.25 & 1149593 \\
30 & 0.51 & -0.93 & -0.41 & 0.26 & 172064 \\
31 & 0.16 & -0.13 & 0.03 & 0.12 & 322522 \\
32 & 0.10 & 0.00 & 0.10 & 0.16 & 330994 \\
33 & -0.21 & 0.28 & 0.07 & 0.21 & 716940 \\
34 & -0.23 & -0.11 & -0.34 & 0.15 & 1089663 \\
35 & -0.78 & 0.21 & -0.57 & 0.28 & 1318406 \\
36 & -0.99 & 0.12 & -0.88 & 0.43 & 1980369
\end{tabular}

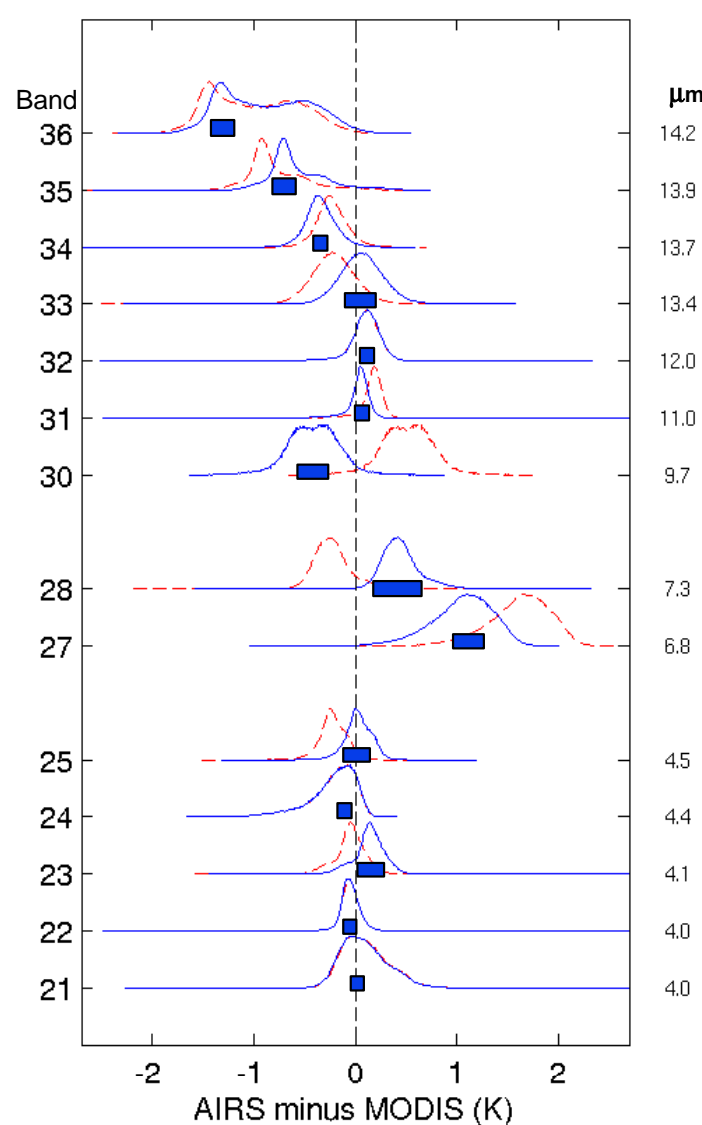

AIRS minus MODIS $(\mathrm{K})$

Figure 11. Distributions of brightness-temperature differences between AIRS and MODIS for a large number of samples (N) taken from scenes with reasonably small spatial non-uniformity. An spectral shift in the MODIS spectral response functions could account for most of these differences. It has been demonstrated that a single spectral shift would make the differences small for a wide range of scene brightness temperature conditions.

\section{Summary}

These initial results of comparing AIRS in orbit on the Aqua platform to aircraft observations from S-HIS illustrate the substantial advantages of high spectral resolution observations for accurate calibration applications.

The basic conclusions are listed below:

1. The calibration uncertainty of advanced high spectral resolution infrared observations are approaching the $0.1 \mathrm{~K}$ desired for climate applications,

2. High spectral resolution observations from airborne instruments (S-HIS \& NAST) are now proven tools for the detailed validation of satellite based observations,

3. AIRS is providing high quality global radiances for atmospheric sounding \& climate applications, and a calibration reference for other IR instruments, and

4. High spectral resolution Aircraft comparisons provide a way to periodically test the absolute calibration of spacecraft instruments with instrumentation that can be carefully re-calibrated with reference standards on the ground, which is especially valuable for assuring the long-term consistency and accuracy of weather and climate observations. 


\section{Acknowledgements}

We gratefully acknowledge the support of the Integrated Project Office (IPO), contract 50-SPNA-1-00039 and of NASA contract NAS5-31375 for support of S-HIS instrument refinement, field deployment, and data analysis for this work. Integration of S-HIS to the Proteus and early development was supported by the DOE ARM Program.

\section{References}

Aumann, H.H., Chahine, M.T., C. Gautier, M.D. Goldberg, E. Kalnay, L.M. McMillan, H. Revercomb, P.W. Rosenkranz, W.L. Smith, D.H. Staelin, L.L. Strow, and J. Susskind, 2003: AIRS/AMSU/HSB on the Aqua Mission: Design, Science Objectives, Data Products, and Processing Systems. IEEE Transactions on Geoscience and Remote Sensing, 41, p 253-264.

Best, F.A.; Revercomb, H.E.; LaPorte, D.D.; Knuteson, R.O., and Smith, W.L, 1997: Accurately calibrated airborne and ground-based Fourier transform spectrometers II: HIS and AERI calibration techniques, traceability, and testing. In: Proceedings of the Council for Optical Radiation measurements (CORM) 1997 Annual Meeting, National Institute of Standards and Technology (NIST), Gaithersburg, MD

Revercomb, H.E., D.D. LaPorte, W.L. Smith, H. Buijs, D.G. Murcray, F.J. Murcray, and L.A. Sromovsky, 1988a: High-Altitude Aircraft Measurements of Upwelling IR Radiance: Prelude to FTIR from Geosynchronous Satellite. Mikrochimica Acta [Wien], II, 439-444.

Revercomb, H.E., H. Buijs, H.B. Howell, D.D. LaPorte, W.L. Smith, and L.A. Sromovsky, 1988b: Radiometric Calibration of IR Fourier Transform Spectrometers: Solution to a Problem with the High Resolution Interferometer Sounder. Applied Optics, 27, 3210-3218.

Revercomb, H.E., H. Buijs, H.B. Howell, R.O. Knuteson, D.D. LaPorte, W.L. Smith, L.A. Sromovsky, and H.W. Woolf, 1989: Radiometric Calibration of IR Interferometers: Experience from the High-resolution Interferometer Sounder (HIS) Aircraft Instrument. RSRM '87:Advances in Remote Sensing Retrieval Methods, A. Deepak, H. Fleming, J. Theon (Eds.). A. Deepak Publishing, Hampton, Virginia

Revercomb, H. E., 1994: Techniques for Avoiding Phase and Non-linearity Errors in Radiometric Calibration: A Review of Experience with the Airborne HIS and Ground-based AERI. Keynote Address, Proceedings of the 5th International Workshop on Atmospheric Science from Space using FTS, p 353378, Tokyo, Japan, 30 November -2 December.

Revercomb, H. E., W. L. Smith, F. A. Best, J. Giroux, D. D. LaPorte, R. O. Knuteson, M. W. Werner, J. R. Anderson, N. N. Ciganovich, R. W. Cline, S. D. Ellington, R. G. Dedecker, T. P. Dirkx, R. K. Garcia, and H. B. Howell, 1996: Airborne and ground-based Fourier transform spectrometers for meteorology: HIS, AERI and the new AERI-UAV. Proceedings SPIE Optical Instruments for Weather Forecasting, edited by G.W. Kamerman, 2832, 106-117.

Revercomb, H. E., F. Best, D. LaPorte, R. Knuteson, W. Smith, N. Ciganovich, R. Dedecker, T. Dirkx, R. Garcia, R. Herbsleb, J. Short, and H. Howell, 1997: Accurately Calibrated Airborne and Ground-based Fourier Transform Spectrometers I: HIS and AERI Instrument Design, Performance, and Applications for Meteorology and Climate. Council for Optical Radiation Measurements (CORM) 1997 Annual Meeting, National Institute of Standards and Technology (NIST), Gaithersburg, Maryland, 29 April.

Revercomb, H.E., D. C. Tobin, V.P. Walden, J. Anderson, F.A. Best, N.C. Ciganovich, R.G. Dedecker, T. Dirkx, S.C. Ellington, R.K. Garcia, R. Herbsleb, H.B. Howell, R.O. Knuteson, D. LaPorte, D. McRae, and M. Werner, Recent Results from Two New Aircraft-based Instruments: the Scanning Highresolution Interferometer Sounder (S-HIS) and the NPOESS Atmospheric Sounder TestbedInterferometer (NAST-I), Proceedings of the Eighth International Workshop on Atmospheric Science from Space using Fourier Transform Spectrometry (ASSFTS8), Toulouse, France, 16-18 November, 1998; sponsored by Meteo-France, CNES, CNRS; p 249-254.

Smith, W.L., H.M. Woolf, H.B. Howell, H.-L. Huang, and H.E. Revercomb, 1989: The Simultaneous Retrieval of Atmospheric Temperature and Water Vapor Profiles - Application to Measurements with the High-resolution Interferometer Sounder (HIS). RSRM '87:Advances in Remote Sensing Retrieval Methods, A. Deepak, H. Fleming, J. Theon (Eds.). A. Deepak Publishing, Hampton, Virginia. 